\title{
Letter: Phrenic Nerve Palsy Secondary to Parsonage-Turner Syndrome
}

\author{
Jose David Avila ${ }^{1}$
}

Received: 17 February 2017 / Accepted: 20 February 2017 / Published online: 4 March 2017

(c) Springer Science+Business Media New York 2017

\section{Dear Editor,}

I read with interest the article by McEnery et al. [1]. They highlight the importance of considering neuralgic amyotrophy (NA) in the evaluation of phrenic neuropathy (PN) and correctly state that this diagnosis is often overlooked. Further, they precisely call for conservative management as the condition frequently carries a good prognosis. I would like to offer 2 additional points.

The authors describe four male patients with dyspnea preceded by shoulder pain. Three of them had electrodiagnostic evidence of unilateral PN and, in the remaining one (case 2), the phrenic motor response was unobtainable on either side but there were radiologic signs of hemidiaphragmatic paralysis. All patients are said not to have other neurologic deficits by history or examination. Additional details regarding the electrodiagnostic studies are not provided and it is unclear if other motor nerves or myotomes were involved.

If the patients had isolated PN, they could be characterized as having probable NA at best [2]. On the other hand, it has been proposed that isolated PN may represent a separate entity in the spectrum of immune-mediated focal neuropathies, rather than a subtype of NA [3]. Even though differentiating between the two may not have an impact on management and prognosis, the distinction may become important for future research as we expand our understanding on the selective involvement of particular nerves in each condition.

Jose David Avila

avila.jd83@gmail.com

1 Department of Neurology, Geisinger Medical Center, 100 N. Academy Ave., Danville, PA 17822, USA
Also, as the authors point out, the association between NA and PN is relatively rare. However, it may be more common in certain groups. In the last few years, NA has been recognized as an extra-hepatic manifestation of hepatitis E. Our recent review of all published cases demonstrated that PN was present in up to $16 \%$ of cases, which is more than twice the proportion reported for typical NA [4]. Although there is no mention of gastrointestinal symptoms in the cases described by McEnery et al., hepatitis E is often mild or subclinical. Testing liver enzymes is a reasonable screening method in these patients.

Funding This study was not supported by grant funding.

Compliance with Ethical Standards

Conflict of interest The author has no conflict of interest to declare.

\section{References}

1. McEnery T, Walsh R, Burke C, McGowan A, Faul J, Cormican L (2017) Phrenic nerve palsy secondary to parsonage-turner syndrome: a diagnosis commonly overlooked. Lung. doi:10.1007/ s00408-016-9972-2

2. Tsao BE, Ostrovskiy DA, Wilbourn AJ, Shields RW (2006) Phrenic neuropathy due to neuralgic amyotrophy. Neurology 66:1582-1584

3. Podnar S (2015) Nosology of idiopathic phrenic neuropathies. J Neurol 262:558-562

4. Avila JD, Lacomis D, Lam EM (2016) Neuralgic Amyotrophy associated with hepatitis $E$ virus infection: first case in the United States. J Clin Neuromuscul Dis 18:96-100 